\title{
Proapoptotic and antiinvasive activity of Racl small molecule inhibitors on malignant glioma cells
}

This article was published in the following Dove Press journal:

OncoTargets and Therapy

30 October 2014

Number of times this article has been viewed

\section{Georgina A Cardama' \\ Nazareno Gonzalez' \\ Matias Ciarlantini ${ }^{2}$ \\ Lucia Gandolfi Donadío \\ María Julieta Comin ${ }^{2}$ \\ Daniel F Alonso' \\ Pablo Lorenzano Mennal,* \\ Daniel E Gomez ${ }^{1, *}$}

'Laboratory of Molecular Oncology, National University of Quilmes, Buenos Aires, Argentina; ${ }^{2}$ Laboratory of Organic Synthesis, Center of Research and Development in Chemistry, National Institute of Industrial Technology, San Martín, Argentina

*These authors contributed equally to this work
Correspondence: Daniel E Gomez Laboratorio de Oncología Molecular, Universidad Nacional de Quilmes, Roque Sáenz Peña 352, Bernal BI876BXD,

Buenos Aires, Argentina

Tel +54 II 43657100

Email degomez@unq.edu.ar
Abstract: Malignant gliomas are characterized by an intrinsic ability to invade diffusely throughout the normal brain tissue. This feature contributes mainly to the failure of existing therapies. Deregulation of small GTPases signaling, in particular Rac1 activity, plays a key role in the invasive phenotype of gliomas. Here we report the effect of ZINC69391, a specific Rac1 inhibitor developed by our group, on human glioma cell lines LN229 and U-87 MG. ZINC69391 is able to interfere with the interaction of Rac1 with Dock180, a relevant Rac1 activator in glioma invasion, and to reduce Rac1-GTP levels. The kinase Pak1, a downstream effector of Dock180-Rac1 signaling, was also downregulated upon ZINC69391 treatment. ZINC69391 reduced cell proliferation, arrested cells in G1 phase, and triggered apoptosis in glioma cells. Importantly, ZINC69391 dramatically affected cell migration and invasion in vitro, interfering with actin cytoskeleton dynamics. We also evaluated the effect of analog 1A-116, a compound derived from ZINC69391 structure. 1A-116 showed an improved antiproliferative and antiinvasive activity on glioma cells. These findings encourage further preclinical testing in clinically relevant animal models.

Keywords: GTPases. invasion, Dock180, small molecule

\section{Introduction}

Gliomas are brain neoplasms with a glial cell origin and represent the most common primary tumors of the central nervous system in humans, with a patient mean survival of less than 1 year. The current treatment for gliomas includes the use of surgery, radio-, and chemotherapy. ${ }^{1}$ Despite their limited metastatic potential outside the central nervous system, gliomas are characterized by their high invasion potential throughout the brain. Migration and invasion of glioma cells is one of the most challenging processes that mainly contributes to the high frequency of tumor recurrence and tumor progression. ${ }^{2}$ Consequently, new therapeutic approaches are essential to improve treatment outcome.

A key group of molecules involved in glioma cell invasion are Rho GTPases. These proteins are molecular switches that cycle between two conformational states: an inactive GDP-bound form and an active GTP-bound form. This cycle is highly regulated by guanine nucleotide exchange factors (GEFs) that catalyze nucleotide exchange and mediate activation and GTPase-activating proteins that stimulate GTP hydrolysis and inactivate the GTPase. ${ }^{3,4}$ The active GTP-bound state binds preferentially to downstream effector proteins, such as the p-21 activated kinase (Pak) family of proteins, and actively transduces signals. ${ }^{5}$ 
Rac1 is one of the most studied members of the Rho GTPases family and controls fundamental cellular processes. Rac1 is a major regulator in actin cytoskeleton reorganization, affecting endocytosis and trafficking, cell cycle progression, and cell adhesion and migration. Accumulating evidence indicates that Rac 1 is overexpressed and/or hyperactivated in a wide range of tumors. ${ }^{6}$ In astrocytomas, Rac1 is overexpressed in high-grade tumors. Notably, the plasma membrane localization of Rac1 observed in a subset of glioblastoma multiforme tumors, but not in low-grade astrocytomas or non-neoplastic brain, indicates that Rac1 is hyperactivated in glioblastomas. ${ }^{7}$ Importantly, Rac1 was identified as a key molecule involved in the invasive behavior of gliomas. Many GEFs have been found to contribute to Rac1 activation and mediate glioma cell motility, such as Ect2, Vav3, Trio, and Dock180, among others. ${ }^{7,8}$

Due to their key role in many transforming events in different cancer types, Rho GTPases are therefore attractive and validated targets for anticancer therapies. ${ }^{9}$ Recently, we identified ZINC69391 as a small molecule that inhibited Rac1-GEF interaction and was able to affect cell proliferation, cell cycle progression, and migration of highly aggressive breast cancer cell lines. Moreover, ZINC69391 inhibited lung metastasis in vivo. We further developed novel analogs using ZINC69391 as the parental compound and we showed that $1 \mathrm{~A}-116$ analog is a more potent Rac1 inhibitor in vitro and in vivo. Both ZINC69391 and 1A-116 had no effect on Cdc42 GTPase activation, a protein closely related to Rac1 GTPase. $^{10}$

Here, we report the biological activity of ZINC69391 on glioblastoma cells, showing a reduction of cancer cell growth associated with an elevated cell death, and a decrease in glioma cell migration and invasion in vitro. In line with our previous results, 1A-116 also showed to be a more potent agent on glioma cells.

\section{A}

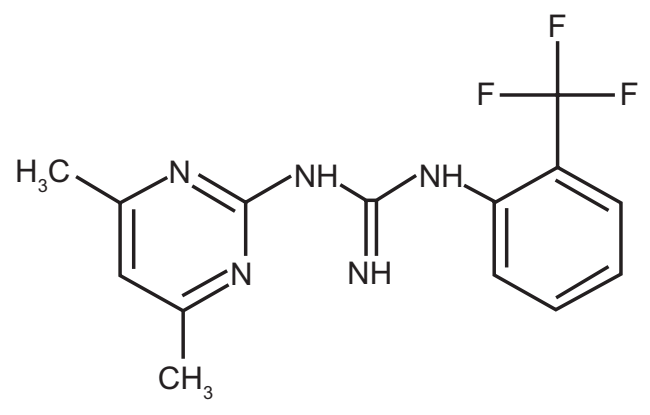

\section{Materials and methods}

\section{Cell lines}

Human glioblastoma multiforme cell lines LN229 (ATCC CRL-2611) and U-87 MG (ATCC HTB-14) were obtained from ATCC. Cells were grown in Dulbecco's Modified Eagle's Medium (DMEM) or Minimum Essential Media (Thermo Fisher Scientific, Waltham, MA, USA), respectively, supplemented with $10 \%$ heat-inactivated fetal bovine serum, $2 \mathrm{mM}$ glutamine, and $80 \mu \mathrm{g} / \mathrm{mL}$ gentamicin at $37^{\circ} \mathrm{C}$ in $5 \%$ $\mathrm{CO}_{2}$ atmosphere. Cell cultures were routinely subcultured twice a week by trypsinization using standard procedures.

\section{Compounds}

ZINC69391 (shown in Figure 1A) and 1A-116 (shown in Figure 1B) were synthesized as reported ${ }^{10}$ in Laboratorio de Síntesis Orgánica, Centro de Química, Instituto Nacional de Tecnología Industrial (INTI). Depending on the setting, concentrations between 10 and $100 \mu \mathrm{M}$ were used as described in each experiment. Structures are shown in Figure 1.

\section{Cell proliferation assay}

$2.5 \times 10^{4}$ cells were plated in 96-well plates and then treated with different compounds for 72 hours. Cell growth was measured by colorimetric MTT assay (Sigma-Aldrich Co, St Louis, MO, USA).

The sequences of small interfering RNA (siRNA) for the sense and antisense strands were: for anti-Rac1 siRNA, sense 5'- GUU CUU AAU UUG CUU UUC CTT - $3^{\prime}$ and antisense 3'-TTC AAG AAU UAA ACG AAA AGG-5' were described elsewhere; 11 and for control siRNA, sense $5^{\prime}$-CAG UCA GGA GGA UCC AAA GTT-3' and antisense 3'-TTG UCA GUC CUC CUA GGU UUC-5' as described elsewhere. ${ }^{12}$ Synthetic oligonucleotides prepared by Thermo Fisher Scientific were annealed to form a short double-stranded RNA with a 3'-dithymidine overhang. On day 0, LN229 cells were plated on $60 \mathrm{~mm}$ dishes, grown until approximately

B

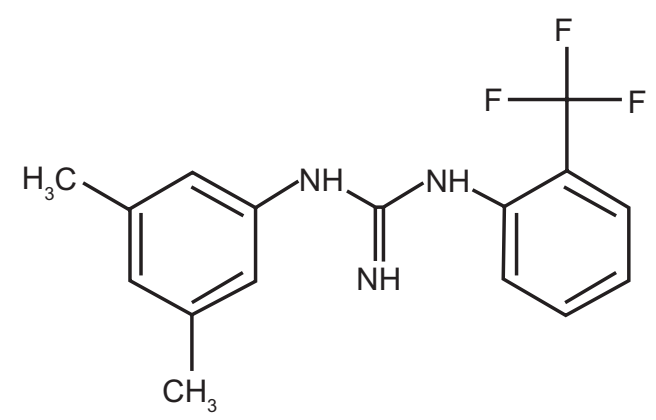

Figure I Chemical structures of Racl inhibitors.

Notes: (A) Chemical structure of ZINC6939I (CI4HI5F3N5; molecular weight, 310.303). (B) Chemical structure of IA-II6 analog (CI6HI6F3N3; molecular weight, 307.3I). 
$30 \%-50 \%$ confluency, and then transfected with Rac1 siRNA or control siRNA using Lipofectamine 2000 (Thermo Fisher Scientific), following manufacturer's instructions. After 4 days, cells were submitted to a second round of transfection under the same conditions. On day $7,2.5 \times 10^{4}$ cells were plated in 96-well plates and then treated with different concentrations of 1A-116 for 72 hours. Cell growth was measured by colorimetric MTT assay. Results shown correspond to the average of three separate experiments.

\section{Western blot}

Cells were treated and lysed in lysis buffer. Cell lysates were clarified and quantified by Bradford. Equal amounts of total protein for each condition were boiled for 5 minutes in $4 \mathrm{X}$ sample buffer and resolved by $12 \%$ SDS-PAGE. Samples were then transferred to PVDF membranes (HybondP; Amersham Biosciences) and analyzed by Western blotting using anti-Pak1 (Millipore) and anti-phosphor-Pak1 (Millipore). Secondary antibody binding was detected by enhanced chemiluminescence.

\section{Affinity precipitation of Racl-Dock I 80}

For Rac1-Dock180 precipitation assay, LN229 cells that express high endogenous levels of Dock180 were lysed in EDTA buffer (25 mM Tris, pH 7.5, $150 \mathrm{mM} \mathrm{NaCl}$, $5 \mathrm{mM}$ ethylenediaminetetraacetic acid, $0.5 \%$ NP-40, $5 \mathrm{mM}$ $\beta$-glycerophosphate) supplemented with protease inhibitor cocktail. Lysates were cleared and $400 \mu \mathrm{g}$ of total protein was used per condition. Glutathione Sepharose 4B beads (GE Healthcare) coupled with bacterially expressed glutathione S-transferase (GST)-Rac1 were preincubated with different concentrations of ZINC69391 for 30 or 15 minutes at $4{ }^{\circ} \mathrm{C}$ and then added to each protein extract in the presence or absence of ZINC69391, using vehicle as control. The beads were washed twice with lysis buffer, resuspended in sample buffer, and boiled. The samples were analyzed by Western blot using mouse monoclonal anti-Dock180 antibody (Santa Cruz Biotechnology Inc., Dallas, TX, USA). The integrity of the purified fusion proteins was checked by SDS-PAGE and Coomassie blue staining prior to use in the assays described below.

\section{Racl pulldown assay}

Tumor cells were plated at a density of $2.5 \times 10^{5}$ cells/well in six-well tissue plates, grown to $80 \%$ confluence, and starved for 48 hours. The cells were then treated for 1 hour with the compound and stimulated for 15 minutes with epidermal growth factor (EGF) $100 \mathrm{ng} / \mathrm{mL}$ (Thermo Fisher Scientific). Monolayers were washed with phosphate-buffered saline
(PBS) and lysed in 150-GPLB buffer (20 mM Tris, pH 7.4, $150 \mathrm{mM} \mathrm{NaCl}, 5 \mathrm{mM} \mathrm{MgCl}, 0.5 \%$ NP-40, 10\% glycerol, $\mathrm{pH}$ 7.4) supplemented with a protease inhibitor cocktail (Sigma-Aldrich Co). Lysates were clarified and the protein concentrations were normalized. An aliquot was removed for determination of total Rac1 and the rest was incubated with Glutathione Sepharose 4B Beads (GE Healthcare) coupled with bacterially expressed GST-Pak. Bound complexes were washed with lysis buffer, resuspended in protein sample buffer, boiled, and loaded onto a 12\% SDS-PAGE gel. Proteins were transferred and blotted with mouse monoclonal antibody against Rac1 (Sigma-Aldrich Co).

\section{Cell cycle analysis}

For cell cycle analysis by flow cytometry, cells were washed and incubated in serum-free DMEM for synchronization for 24 hours. Cells were then treated for 48 hours with different concentrations of ZINC69391 in DMEM supplemented with $10 \%$ fetal bovine serum and collected by trypsinization. Cells were fixed in 70\% methanol in PBS and stained with propidium bromide ( $1 \mathrm{mg} / \mathrm{mL}$ ) (Thermo Fisher Scientific). Cell cycle progression was analyzed in a BD FACSCalibur ${ }^{\mathrm{TM}}$ (BD Biosciences, San Jose, CA, USA) flow cytometer. Before recording 10,000 events, the verification of the doublet discrimination function of the flow cytometer was performed with a DNA QC Particles kit (BD Biosciences).

\section{Actin staining}

Cells grown in glass cover slips were incubated overnight (16 hours) in serum-free DMEM, treated for 1 hour with ZINC69391, and then stimulated with EGF (100 ng/mL) (Thermo Fisher Scientific) for 15 minutes. Cells were fixed in 4\% formaldehyde in PBS and stained with AlexaFluor555 conjugated phalloidin (Molecular Probes; Thermo Fisher Scientific) following the manufacturer's instructions. Images were recorded in an inverted fluorescence microscope (Nikon Eclipse T2000; Nikon Corporation, Tokyo, Japan).

\section{Transwell migration assay}

Cells were starved overnight in DMEM media, and $1 \times 10^{5}$ cells were preincubated with or without ZINC69391 for 30 minutes and then added to the top chambers of 24-well transwell plates (Nunc; $8 \mu \mathrm{m}$ pore size). Media with $10 \%$ FCS was added to the bottom chambers. After overnight incubation, top (nonmigrating) cells were removed and bottom (migrating) cells were fixed and stained with crystal violet. The number of migrating cells in five fields was counted. The experiments were repeated a minimum of three times. 


\section{Transwell invasion assay}

Transwell chambers were coated with $100 \mu \mathrm{L}$ of a $0.8 \mathrm{mg} / \mathrm{mL}$ Matrigel suspension and dried at room temperature. Using $1.5 \times 10^{5}$ cells and 48 hours' incubation, the assay was conducted as for the migration assay.

\section{Apoptosis}

For annexin V early apoptosis determination, Annexin V-Alexa488/Cell dead apoptosis kit (Molecular Probes; Thermo Fisher Scientific) was used. Cells growing in glass cover slips were treated for 6 hours with $10 \mu \mathrm{M}$ and $50 \mu \mathrm{M}$ ZINC69391 and then stained according to manufacturer's protocol. For terminal deoxynucleotidyl transferase dUTP nick end labeling (TUNEL) assay, DeadEnd Fluorometric TUNEL System (Promega Corporation, Fitchburg, WI, USA) was used. Apoptosis was determined using manufacturer's protocol using the same experimental setting described for Annexin V Apoptosis determination.

\section{Results}

\section{ZINC6939I inhibited Racl interaction} with Dock I80, a relevant GEF in glioma biology

ZINC69391 was developed as a Rac1 activation inhibitor, interfering with Rac1-GEF interaction by masking Trp56 residue on Rac1 surface. Dock180 belongs to the atypical family of GEFs and has been shown to have an important role on glioma cell migration. ${ }^{8}$ The Dock family of GEFs show little homology to the classical Dbl family members, but it has been shown that Trp56 residue on Rac1 is a key residue for specific Dock180-Rac1 recognition. ${ }^{13}$ Thus, we determined the effect of ZINC69391 treatment on Rac1-Dock180 interaction. Because it was reported that LN229 expresses high levels of Dock180, ${ }^{8}$ this cell line was used to assess Rac1-Dock180 interaction. LN229 lysates were incubated with GST-Rac1 coupled with glutathione agarose beads in the presence or absence of ZINC69391. As seen in Figure 2A, ZINC69391 was able to block Rac1-Dock180 interaction in vitro starting at $50 \mu \mathrm{M}$, showing that ZINC69391 is also able to block Rac1 interaction with the atypical family of GEFs.

\section{ZINC6939I inhibited EGF dependent Racl activation and downstream phosphorylation of Pakl in a concentration-dependent manner}

We next tested the ability of ZINC69391 to reduce Rac1 activation on LN229 cells. LN229 were serum-starved overnight, treated for 1 hour, and then stimulated with EGF. The cell lysates were subjected to pulldown assays and Rac1GTP was determined using a Rac1 monoclonal antibody. Figure 2B shows that treatment with ZINC69391, prior to EGF stimuli, dramatically impaired Rac1 pathway activation by this growth factor in a concentration-dependent manner; whereas total Rac1 levels remained unchanged. Remarkably, high concentrations of ZINC69391 reduced Rac1 activation below nonstimulated basal levels.

In addition, we tested the effect of ZINC69391 on a relevant Rac1 effector Pak1 that has been shown to play an important role in proliferation, survival, and invasiveness of cancer cells. ${ }^{14}$ More critically, Pak1 phosphorylation in the cytoplasm of cancer cells correlates with shorter survival of glioma patients. ${ }^{15}$ ZINC69391 reduced Pak1 phosphorylation in a concentration-dependent manner using the same experimental scenario as described above. ZINC69391 showed Pak1 phosphorylation inhibition at $10 \mu \mathrm{M}$ in the presence of EGF, but higher concentrations reduced phospho-Pak levels leading to activation levels similar to those found in the unstimulated condition (Figure 2C). This molecular evidence in addition to inhibition of Rac1 activation and of Rac1-Dock180 interaction shows that ZINC69391 is an effective Rac1-axis inhibitor.

\section{ZINC6939I triggered cell cycle arrest and reduced cell proliferation of human glioma cells}

We then analyzed the effect of ZINC69391 on cellular proliferation of glioma cells. After 72 hours' treatment, cell viability was measured using the MTT assay. Treatment of U-87 MG and LN229 cells with ZINC69391 reduced cell proliferation in a concentration-dependent manner (Figure 3A). To further delineate the mechanism of cell-proliferation inhibition, we analyzed the cell cycle distribution after ZINC69391 treatment of LN229 cells. Of interest, Rac1 has been shown to control signal transduction pathways that are essential for cell growth by modulating cell cycle progression through G1 phase through cyclin D1 expression. ${ }^{16}$ As expected, ZINC69391 treatment resulted in a significant increased percentage of cells in the sub-G0/G1 phase (Figure 3B) in a concentration dependent manner.

\section{Racl inhibition by ZINC6939I induced apoptosis on glioma cells}

Since sub-G0 population is often used to estimate apoptosis $^{17}$ and we determined that ZINC69391 treatment 
A

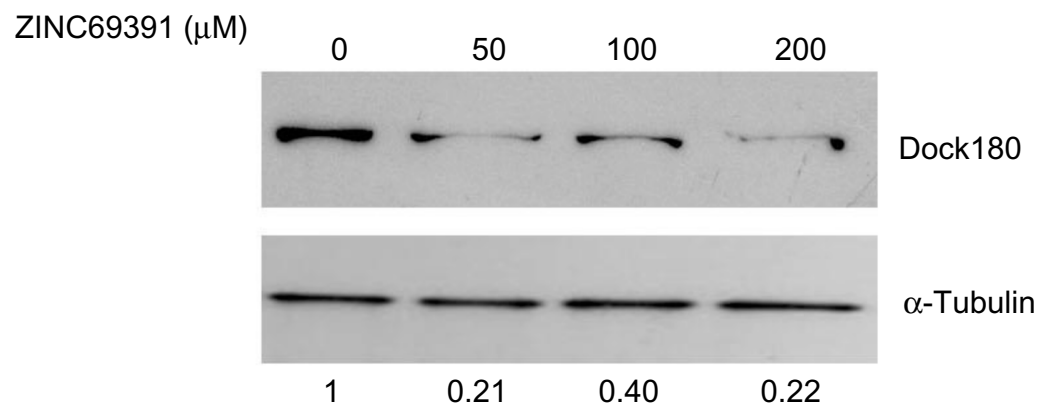

B

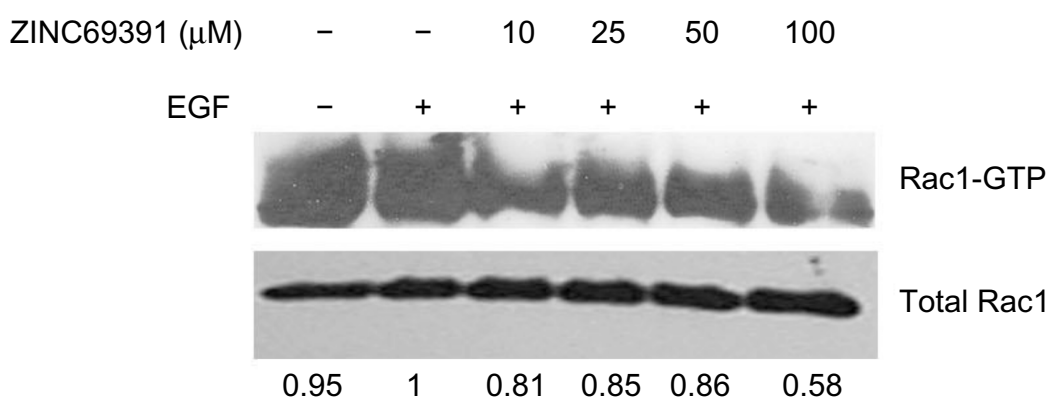

C

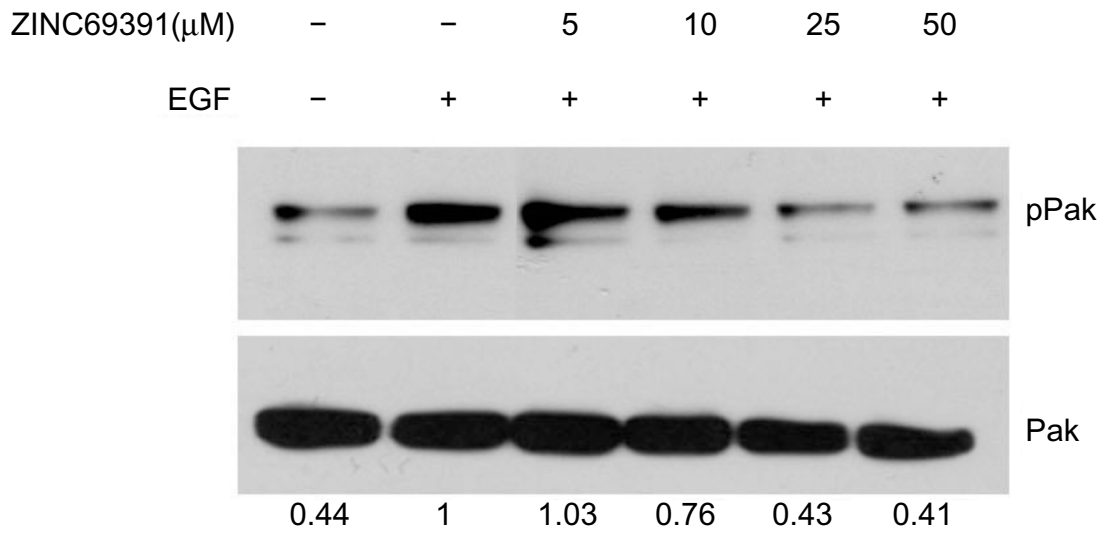

Figure 2 ZINC6939I inhibited Racl activation on LN229 glioma cells.

Notes: (A) Blockade of Racl-Dockl80 interaction. Dockl80 was affinity-precipitated with bacterially expressed Racl immobilized in glutathione agarose beads in the presence of varying concentrations of ZINC6939I. Western blot analysis was carried out with anti-DockI80 antibody. The experiment was repeated three times. Densitometric values are shown below (arbitrary units). (B) Concentration-dependent Racl inhibition by ZINC6939I in glioma cells. Serum-starved LN229 cells were treated for I hour with different ZINC6939I concentrations and stimulated with epidermal growth factor (EGF) (I00 ng/mL) for I5 minutes. Densitometric values are shown below (arbitrary units). (C) Concentration-dependent inhibition of Pakl phosphorylation using the same experimental procedure as before. Densitometric values are shown below (arbitrary units).

results in an increase in this population, we further evaluated the proapoptotic effect of this compound. Rac1 inhibition has long been reported to trigger apoptosis in many different cancer types. Particularly in glioma cells, Rac1 suppression induces apoptosis in cell lines and short-term primary cultures of grade III and grade IV gliomas. ${ }^{18}$

To evaluate whether ZINC69391 induces apoptosis, we used two different techniques. First, we analyzed apoptosis using annexin $\mathrm{V}$, which measures the loss of the phospholipid 
A

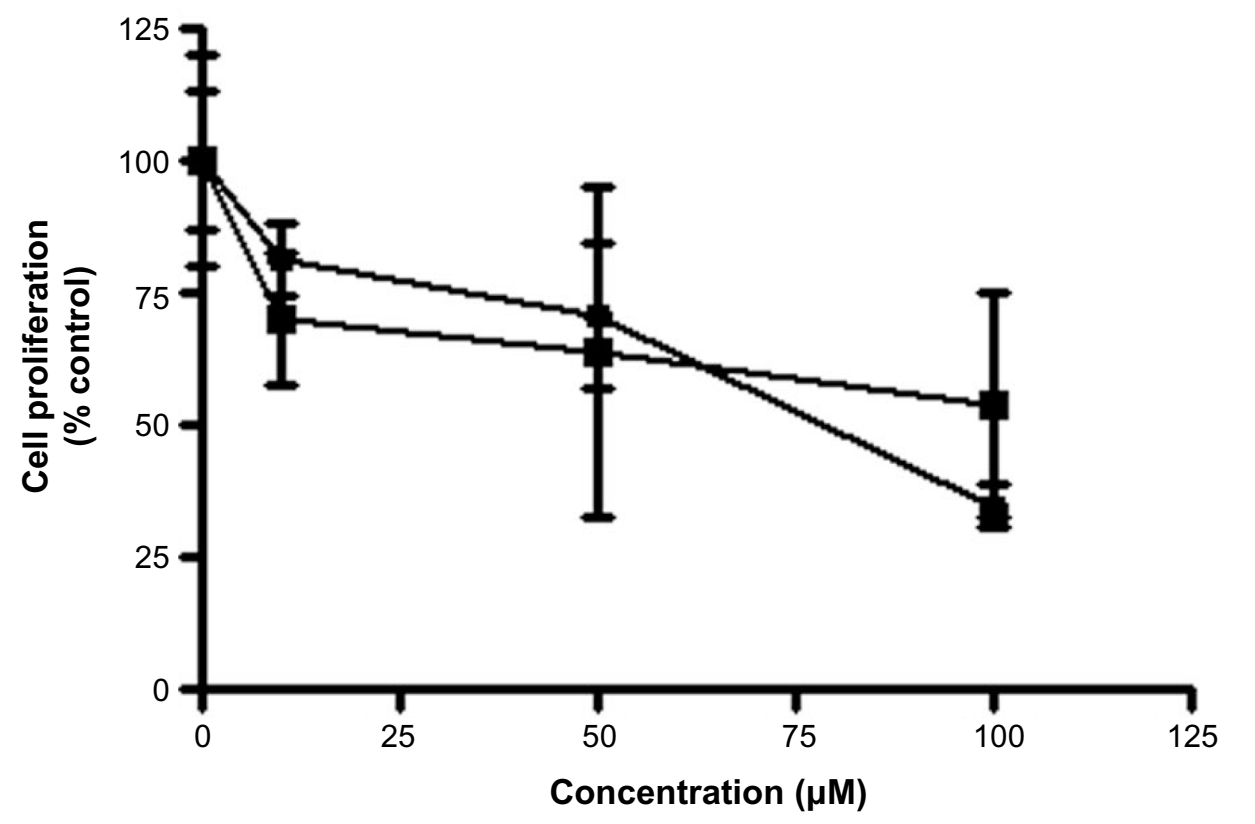

B

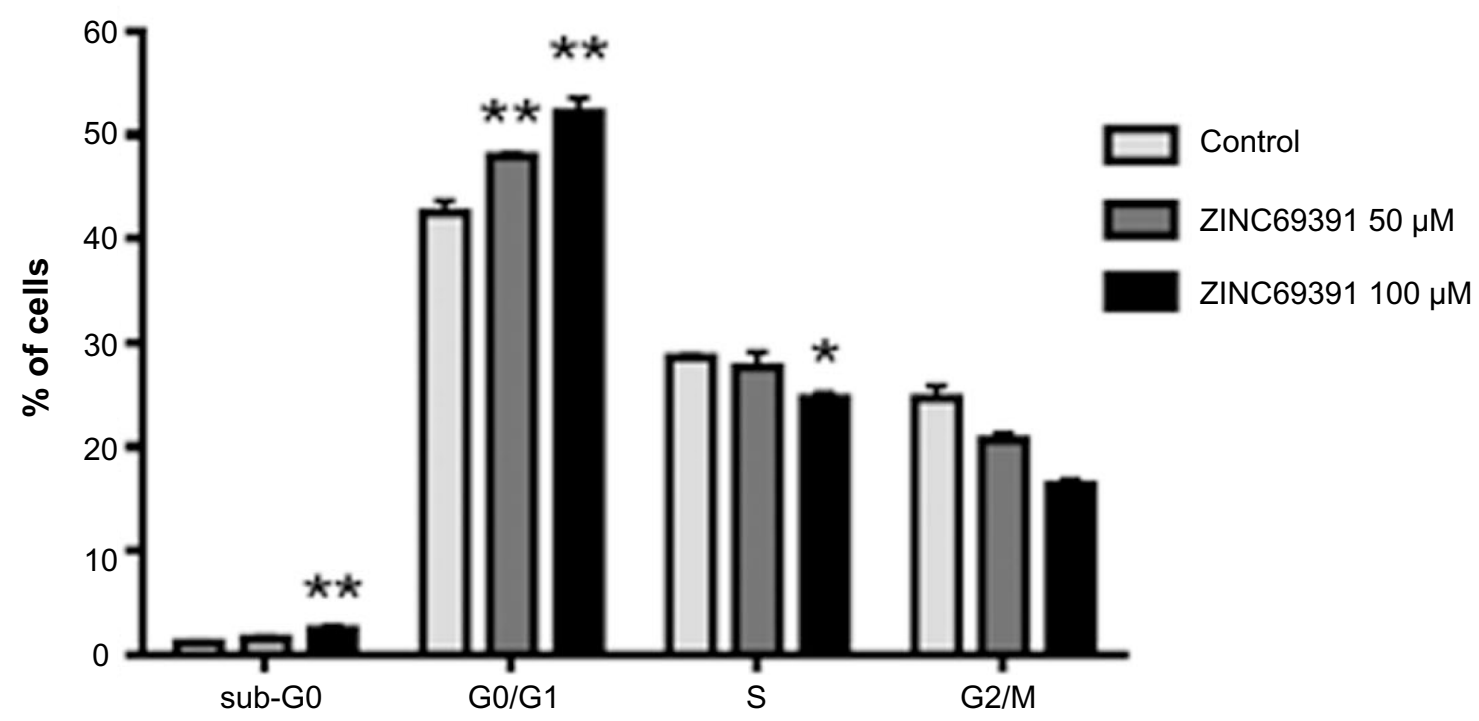

Figure 3 ZINC6939I affected cell proliferation and cell cycle progression of glioma cells.

Notes: (A) ZINC6939I inhibited cell proliferation. LN229 and U-87 MG cells were treated for 72 hours with different concentrations of ZINC6939I. Cell viability was measured using MTT assay. (B) ZINC6939I arrested cell cycle progression in GI phase. LN229 cells were synchronized and treated for 48 hours with ZINC6939I with different concentrations. Cells were fixed, stained with propidium iodide, and analyzed by flow cytometry to estimate the percentage of cells in sub-G0 phase, $\mathrm{GI}$ phase, $\mathrm{S}$ phase, and $\mathrm{G} 2 / \mathrm{M}$ phase. Bars, standard error of the mean. $* P<0.05$, ${ }^{*} * P<0.01$ determined by analysis of variance contrasted. Dunnett's multiple comparison test versus control in each phase.

membrane asymmetry of the cell as an early indicator of apoptosis. In this case, cells were treated for 6 hours with different concentrations of ZINC69391 and then stained with Annexin V-Alexa488 following manufacturer's protocol. As seen in Figure 4A, ZINC69391 was able to trigger apoptosis starting at a concentration of $10 \mu \mathrm{M}$. To further confirm the induction of programmed cell death by ZINC69391, we performed a TUNEL assay, which detects DNA breakage that occurs during late stage of apoptosis. Under the same experimental conditions as before, ZINC69391 presented a significant proapoptotic effect starting at $10 \mu \mathrm{M}$ (Figure 4B and C). In summary, we demonstrated by two different methodologies 
A
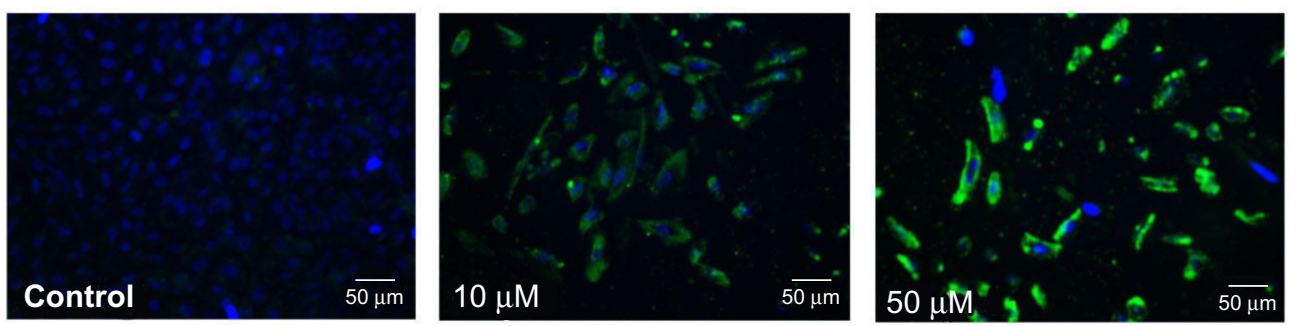

B
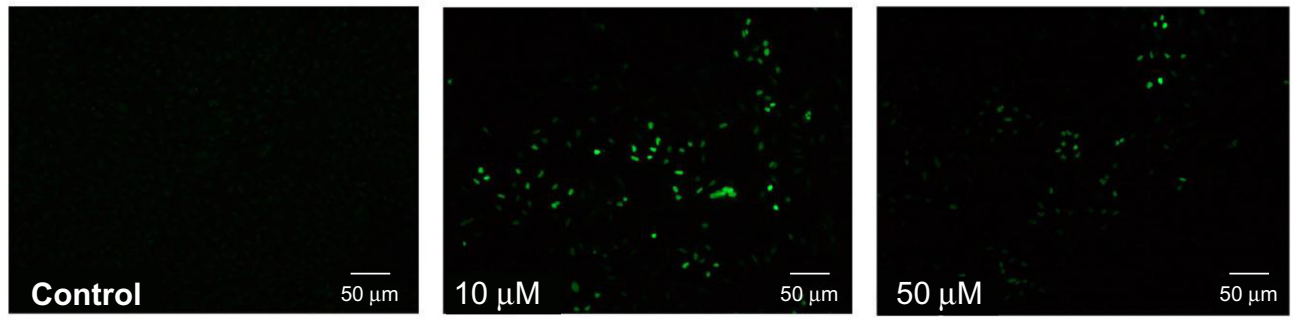

C

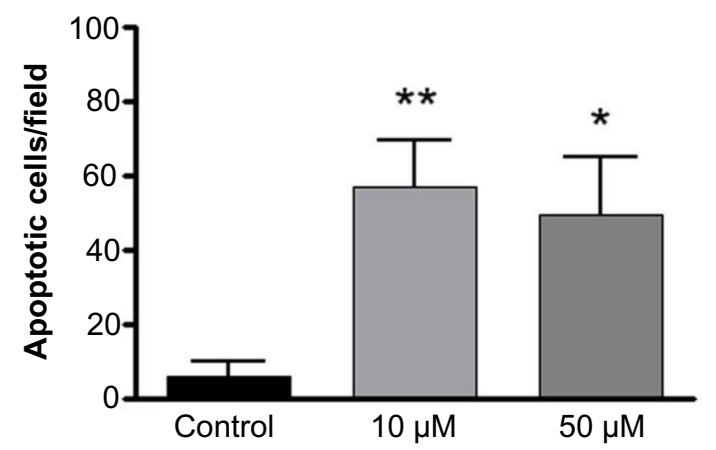

Figure 4 ZINC6939I triggered apoptosis on malignant glioma cells.

Notes: (A) Apoptosis of LN229 cells after $10 \mu \mathrm{M}$ and $50 \mu \mathrm{M}$ treatment for 6 hours using annexin $\mathrm{V}$ staining. Representative micrographs taken at $200 \times$. (B) Late apoptosis was evaluated at $10 \mu \mathrm{M}$ and $50 \mu \mathrm{M}$ for 6 hours using TUNEL assay. Representative micrographs taken at 100×. (C) Quantification of apoptotic cells per field using TUNEL assay. Bars, standard error of the mean, $* P<0.05$, $* * P<0.0$ I determined by analysis of variance cont. Dunnett's multiple comparison test versus control.

that ZINC69391 presents a proapoptotic activity by inhibition Rac1 activation.

\section{ZINC6939I dramatically affected cell migration and invasion of aggressive glioma cells}

Racl plays a critical role in the invasive behavior of gliomas. This GTPase regulates lamellipodia formation, cell migration, and invasion in glioma cells. ${ }^{19}$ Thus, we examined the effect of ZINC69391 on cell migration of LN229 cells in a transwell assay. Treatment of cells with ZINC69391 $10 \mu \mathrm{M}$ for 24 hours significantly reduced cancer cell migration showing a concentration-dependent inhibition (Figure 5A).
Since it is established that mechanisms that regulate cell migration are fundamental to the invasive phenotype of gliomas, ${ }^{2}$ we expected ZINC69391 to be able to reduce cell invasion also. Therefore, we determined the effect of ZINC69391 treatment on the invasive properties of LN229 using a transwell Matrigel invasion assay. Interestingly, ZINC69391 significantly reduced cell invasion in a concentration-dependent manner, starting at $10 \mu \mathrm{M}$ (Figure 5B).

Rho GTPases are key regulators of cytoskeleton reorganization, the mechanism underlying cell motility and invasion processes. ${ }^{20}$ We therefore investigated the effect of ZINC69391 on actin reorganization using phalloidin staining. Since phalloidin stains polymerized actin, we show that 
A
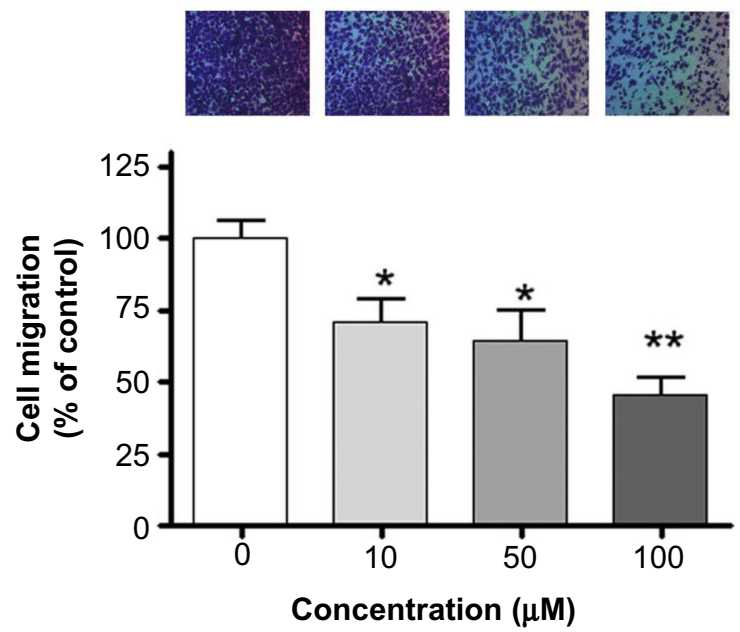

C
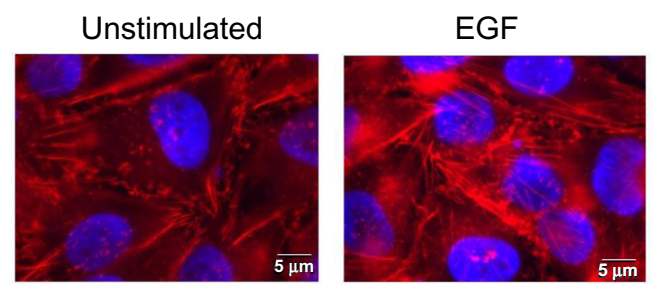

B
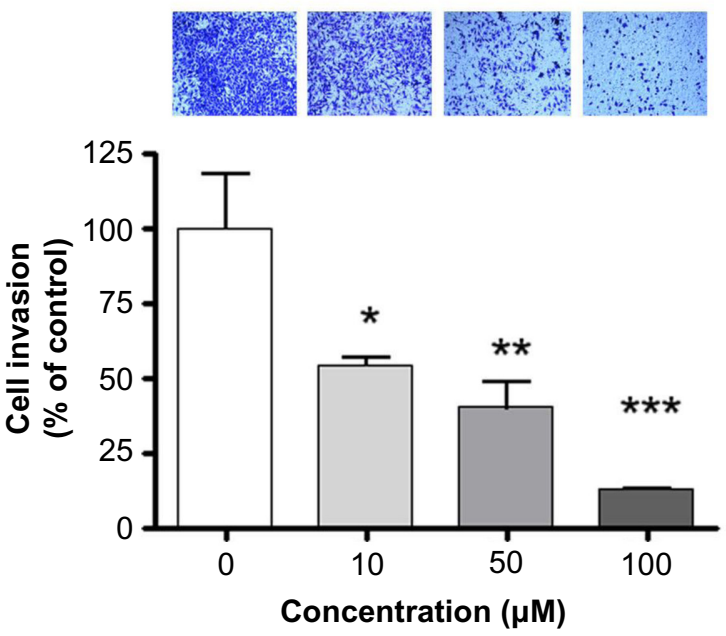

ZINC69391 + EGF

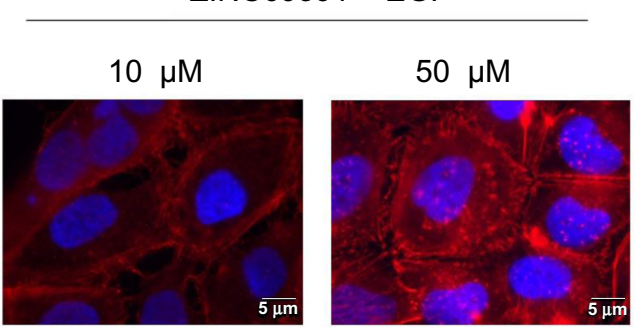

Figure 5 ZINC6939I inhibited glioma cell migration and invasion modulating actin cytoskeleton reorganization.

Notes: (A) LN229 cells were seeded in uncoated transwell chambers with or without ZINC6939I, incubated for I 8 hours, and quantified. Bars, standard error of the mean. $* P<0.05 ; * * * P<0.001$ determined by analysis of variance cont. Dunnett's multiple comparison test. (B) LN229 cells were seeded in Matrigel-coated transwell chambers with or without ZINC6939I, incubated for 48 hours, and quantified. Bars, standard error of the mean. $* P<0.05$; $* * P<0.0$ I; $* * * P<0.00$ I determined by analysis of variance cont. Dunnett's multiple comparison test. (C) Representative micrographs taken at I,000× showing inhibition of epidermal growth factor (EGF)-induced actin reorganization by ZINC6939I in LN229 cells. Cells were grown on cover slips, serum-starved for I6 hours (untreated panel), and treated for I hour with ZINC6939I. After I5 minutes stimulation with EGF (100 ng/mL) (EGF control), cells were fixed and actin filaments were visualized with AlexaFluor555-phalloidin.

ZINC69391 is able to disrupt actin polymerization induced by EGF stimulation. Treatment of cells with $10 \mu \mathrm{M}$ Zinc69391 markedly affected the dynamic reorganization of actin, while with a concentration of $50 \mu \mathrm{M}$ Zinc 69391 the effect is much more evident. ZINC69391 was not able to block completely actin polymerization, but presented a profound effect on actin filaments in the cytoplasm (Figure 5C).

\section{ZINC6939I-derived IA-I I 6 analog presented enhanced Racl-mediated antiproliferative and antiinvasive activity}

We have previously reported the development of a novel analog that showed to be more potent than the parental ZINC69391 compound in aggressive breast cancer cell models in vitro and in vivo. ${ }^{10}$ To evaluate the effect of $1 \mathrm{~A}-116$ on cell proliferation of LN229 and U-87 MG cells, we measured cell viability by the MTT metabolic assay. 1A-116 inhibited cell proliferation in a concentration-dependent manner, as shown in Figure 6A.

To further investigate whether these compounds present a Rac1-mediated antiproliferative effect, we evaluated the effect of 1A-116 on LN229 cells where Rac1 was depleted using siRNA. As shown in Figure 6B, silencing of Rac1 significantly attenuated the inhibitory effects of 1A-116 on cell proliferation compared to control siRNA transfected cells. This result confirms that $1 \mathrm{~A}-116$ affects Rac1 signaling pathway, resulting in a reduction of cell proliferation.

We tested 1A-116 effect on in vitro invasion of LN229 cells by carrying out a transwell Matrigel invasion assay as previously described. Treatment with $50 \mu \mathrm{M} 1 \mathrm{~A}-116$ resulted in a significant reduction of cell invasion, as shown in Figure 6C. Remarkably, 1A-116 analog demonstrated an enhanced antiinvasive activity compared to the parental compound ZINC69391. 
A

LN229

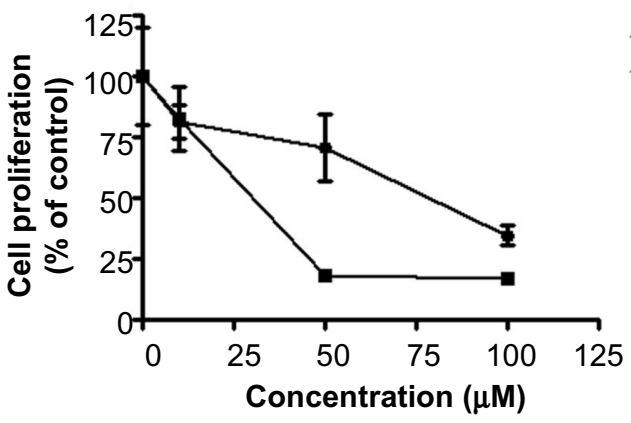

B

LN229

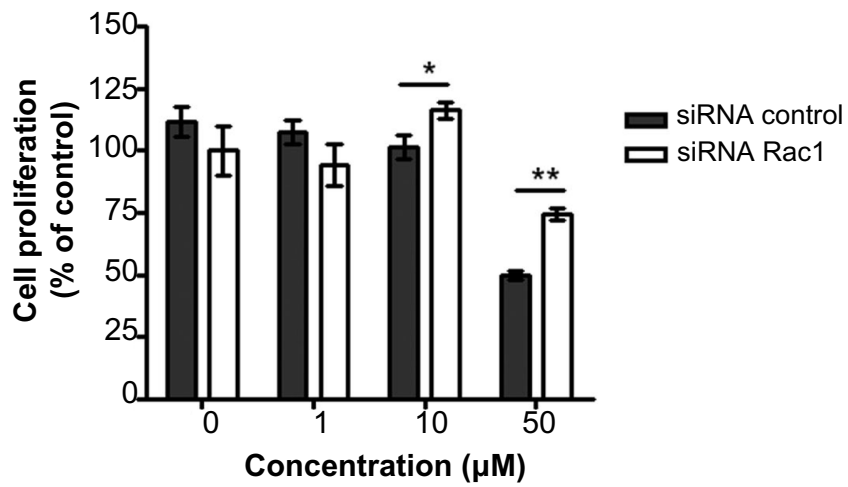

U-87 MG

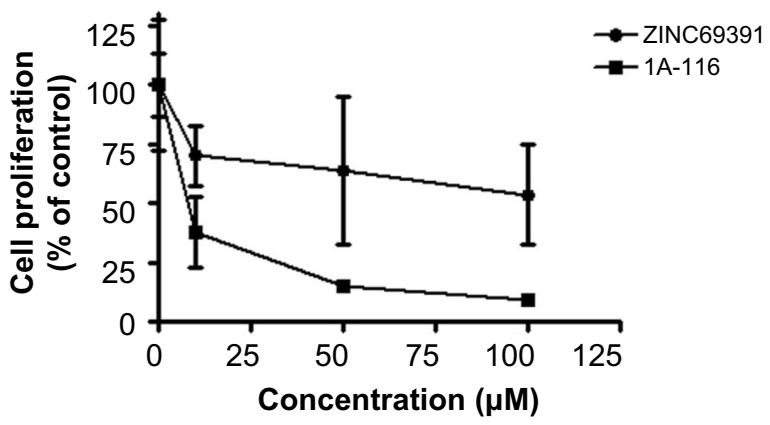

C

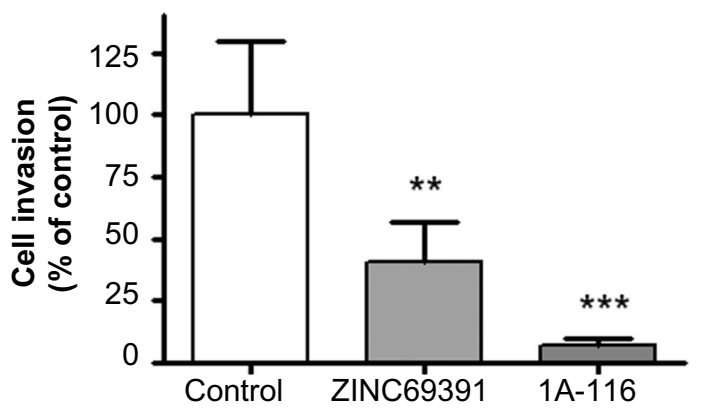

Figure 6 IA- I I6 analog is a more potent Racl inhibitor.

Notes: (A) LN229 and U-87 MG cells were treated for 72 hours with different concentrations of ZINC6939I and IA-II6. Cell viability was measured using MTT assay. (B) LN229 were transiently transfected with Rac I small interfering (si)RNA or control siRNA. Cells were treated for 72 hours with different concentrations of IA-I I6 analog and cell viability was measured using MTT assay. (C) LN229 cells were seeded in Matrigel-coated transwell chambers with or without ZINC6939I, incubated for 48 hours, and quantified. $* * P<0.01$, $* * * P<0.001$. Determined by analysis of variance contrasted with Dunnett's multiple comparison test.

\section{Discussion}

Several studies have showed that deregulated activities of Rho GTPases, such as Rac1, in malignant gliomas are responsible for the highly invasive and infiltrative phenotype of these brain cancers. Although it has recently been described a gain-of-function mutation in racl gene in sunexposed melanomas, ${ }^{21,22}$ to date, these mutations have not been found in other cancer types, including glioblastomas. Despite the fact that activating mutations of $\mathrm{racl}$ were not found, Rac1 protein levels correlate with tumor grade and poor survival in glioblastoma patients. ${ }^{7}$ In addition, immunohistochemistry analyses present a prominent plasma membrane staining of Rac1 in human high-grade glioblastoma specimens, indicating a high activation level of these proteins. This hyperactivation can be explained at least in part by the overexpression of different GEFs, such as Trio, Ect2, Vav3, and Dock180, among others. ${ }^{7}$ Importantly, studies have shown that Rac1 contributes to chemotherapy resistance ${ }^{23}$ and promotes radiation-therapy-induced glioma cell invasion. ${ }^{24}$ Rac1 is also critically involved in the enrichment of the glioma stem-like cell population and tumorigenicity in human glioma. Moreover, Rac1 inhibition on glioma stemlike cells enhanced radiation sensitivity. ${ }^{25}$ Rac1 has also been involved in angiogenesis in different tumoral settings, such as neuroblastoma ${ }^{26}$ and breast cancer. ${ }^{27}$ Inhibition of Rac1 using siRNA reduces vascular endothelial VEGF-mediated tubule formation, migration, and invasion in vitro, and these findings also are seen in vivo. In line with all this, targeting Rac1 activation may be a useful therapeutic strategy for glioblastoma treatment.

In our previous study, we reported the activity of ZINC69391, a novel Rac1 inhibitor with biological activity on aggressive breast cancer cells. ZINC69391 affected breast cancer cell proliferation and migration in vitro and showed a significant reduction by approximately $60 \%$ of total metastatic lung colony formation in a syngeneic animal model. ${ }^{10}$ 
Initially, we searched for molecules capable of binding Rac1 protein surface containing the critical Trp56 residue. Since Trp56 is a key residue in Rac 1 recognition, we expected ZINC69391 may interfere with Rac1 activation by GEFs sharing the same activation mechanism. Two structurally unrelated families of GEFs have been described so far: the classical Dbl and the atypical Dock180-related families. The mechanism involving Trp56 by which many Dbl-GEFs bind and activate Rac1 has been known for more than a decade. ${ }^{28,29}$ More recently, it has been described that Dock180 also shares the same residue as a determinant for specific recognition for Rac1. ${ }^{13}$ In support of this, we have already shown that ZINC69391 was able to interfere with Rac1-Tiam1 (a Dblfamily GEF) recognition and now our results demonstrate that ZINC69391 also blocks Rac1-Dock180 interaction. Dock180 has been characterized to contribute to the enhancement of glioma cell migration and invasion via Rac1 activation. Immunohistochemical analyses on primary human malignant glioma specimens showed that Dock180 is expressed in infiltrating tumor cells within the border and invasive areas compared to the central core of the tumor, independent of tumor grade. On the other hand, Dock180 was not detected in normal brain tissue. ${ }^{8}$ This may have important therapeutic implications, since all malignant gliomas are characterized to invade diffusely, including low-grade astrocytomas. ${ }^{1}$ Dock180 expression may be involved in the early onset of the disease, driving the diffusely infiltrative nature of malignant gliomas. Moreover, Dock180 was found to be involved in the signaling pathways that mediate PDGFR $\alpha$ - and EGFRvIII-driven tumorigenesis and invasion in glioma via Rac1 activation. ${ }^{30,31}$

In line with the evidence indicating that ZINC69391 blocks Rac1-Dock180 interaction, ZINC69391 effectively reduced endogenous Rac1 activation levels, presenting a concentration-dependent inhibition in response to EGF stimulation. Cells treated with ZINC69391 and stimulated with EGF showed Rac1-GTP levels that remained equal or were lower than the unstimulated condition. We also examined the effect of ZINC69391 treatment on Pak1 phosphorylation. Pak1 is a serine/threonine protein kinase that mediates cytoskeletal remodeling and cell motility through actin and microtubules. ${ }^{32}$ Initially, Pak1 was determined to be activated by Rac1 and Cdc42, and to be an important mediator of these GTPases in the brain. ${ }^{33}$ Pak1 is widely expressed in a variety of normal tissues, and its expression is significantly increased in different cancers. ${ }^{34}$ In malignant glioblastoma, immunohistochemical analysis showed that phosphorylated Pak1 levels in the cytoplasm of glioma specimens correlated with shorter survival time in patients. ${ }^{15}$ Our results present that treatment of LN229 cells with ZINC69391 reduced Pak1 phosphorylation in a concentration-dependent manner upon EGF stimulation. Phosphorylated Pak1 levels were found to be lower than the unstimulated condition, when cells were treated with $25 \mu \mathrm{M}$ and $50 \mu \mathrm{M}$ ZINC69391 in the presence of EGF. These results demonstrate that ZINC69391 inhibits Dock180/Rac1/Pak1 signaling pathway on glioma cells in vitro.

In agreement with observations that used expression of dominant-negative Rac1 to inhibit proliferation and survival of glioma cell lines, ${ }^{18,19}$ ZINC69391 treatment showed antiproliferative activity in LN229 and U-87 MG cell lines. This inhibition was associated with a cell cycle arrest in G1 phase. We also showed an increase in sub-G0 cell population, suggesting apoptosis induction. Then, we evaluated the proapoptotic activity of ZINC69391. Annexin V and TUNEL studies established that ZINC69391 induces apoptosis on glioma cell lines at $10 \mu \mathrm{M}$ and $50 \mu \mathrm{M}$, having no effect on cell detachment (data not shown). These findings are in agreement with observations that used recombinant adenoviruses expressing dominant-negative Rac1 on glioma cell lines and glioblastoma primary cultures to suppress Rac1 activity. ${ }^{18}$ Senger et al showed that Rac1 inhibition induces apoptosis in glioma cells but not in normal human astrocytes. These data provide a solid rationale for Rac1 targeting in malignant gliomas, with a selective effect on the tumor. The mechanism by which glioma cells undergo apoptosis upon ZINC69391 treatment remains unknown. Of interest, at least two signaling pathways have been described to contribute to Rac1-mediated survival on glioma cells: JNK and MAPK. The dominant pathway is proposed to be Pak1-JNK pathway. ${ }^{18}$ Here we show a direct effect on Pak1 phosphorylation, but further experiments are necessary to address the effect of ZINC69391 on JNK activity and to determine the pathways and the modulation of different molecules involved in the apoptotic process, such as caspase 3 .

As previously mentioned, diffuse cell migration and invasion are key properties of malignant gliomas. This invasive behavior has major therapeutic implications, since it is the major driver of glioma recurrence and therapeutic failure. ${ }^{1}$ Surgery is unable to cure patients even when the lesions arise in areas in which wide surgical resection is possible, since glioma cells infiltrate throughout the brain. Of interest, several studies have reported that ionizing radiation enhances the invasion of glioma tumor cells. ${ }^{35,36}$ Cancer invasion and drug resistance are thought to be interconnected with the processes promoting tumor progression and therapeutic failure. ${ }^{37}$

Rac1 and Rho GTPases in general have long been known as modulators of cell motility in normal tissues and disease 
tissues. ${ }^{20}$ Particularly in glioblastomas, the spatial regulation of Rho GTPases activity has been studied in detail using biosensors in vivo and in 3D substrates. In both models, glioblastoma cells leading the invasion had higher Rac1 and Cdc42 activities and guided the trailing glioma cells with lower Rac1 and Cdc42 activities. ${ }^{38}$ This differential activation pattern indicates heterogenic invasion potential in different cell populations within the tumor. Importantly, Rac1 GEFs such as Ect2, Vav3, and Trio have been implicated in glioblastoma cell invasion ${ }^{39}$ and Dock180 has been implicated in EGFRvIII-driven invasion. ${ }^{30}$ This shows that Rac1 axis is important for glioma motility. In agreement with observations that used expression of dominantnegative Rac1 and siRNA Rac1 to interfere with glioma cell invasion, our data indicate that ZINC69391 modulates actin cytoskeleton dynamics, inhibiting cell migration and having a significant impact on cell invasion in vitro. Depleting Rac1 on glioma cells using these molecular biology strategies achieved approximately $80 \%$ inhibition of cell invasion. ${ }^{19}$ Our data indicate that modulation of Rac1 by ZINC69391 resulted in approximately $50 \%, 60 \%$, and $85 \%$ cell invasion with $10 \mu \mathrm{M}$, $50 \mu \mathrm{M}$, and $100 \mu \mathrm{M}$ treatment, respectively, leading to similar results compared to dominant-negative experiments using a pharmacological agent.

Our data also show the increased potency of 1A-116 analog compared to parental ZINC69391 compound. This analog was rationally designed using ZINC69391 as a lead structure and was selected from a group of analogs because better docking scores correlated with an improved in vitro and in vivo potency on an aggressive breast cancer model. Importantly ZINC69391 and analog 1A-116 showed to be more potent inhibitors than the described Rac1 inhibitor NSC23766 on cancer cells in vitro $\left(\mathrm{IC}_{50}\right.$ [half maximal inhibitory concentration] values: NSC23766: $140 \mu \mathrm{M}$, ZINC69391: $61 \mu \mathrm{M}, 1 \mathrm{~A}-116: 4 \mu \mathrm{M}) .{ }^{10}$ Interestingly, 1A-116 analog presented also an improved antiproliferative activity compared to the parental analog on glioma cells and this antiproliferative activity showed to be Racl dependent using siRNA probes. These results are in line with previous work, where the overexpression of a constitutively active form of Rac1 (G12V) attenuated the inhibitory effect of 1A-116 analog on breast cancer cells. Together, this demonstrates that the effect of this molecule is mediated by Rac1. We also show that 1A-116 analog is a more potent antiinvasive compound using Matrigel-coated transwells, a key feature for an anticancer agent for glioma treatment.

Because one common obstacle in the treatment of glioblastoma patients is chemoresistance, the need to combine different agents to overcome resistance mechanisms is becoming more evident. In line with this idea, combination schemes with our Rac1 inhibitors and chemotherapeutic drugs used for glioblastoma treatment such as temozolomide may potentially show a therapeutic benefit. Currently we are evaluating these combination schemes. Sooman et al showed by bioinformatics and in vitro studies that camptothecin, a topoisomerase I inhibitor, and imatinib, a tyrosine kinase inhibitor, combined with a Rac1 inhibitor had a synergistic effect on human glioblastoma cell lines. ${ }^{40}$ Karpel-Massler et al also reported that the combination of erlotinib, an EGFR inhibitor, and a Rac1 inhibitor presented a synergistic effect on established glioma cell lines and primary glioblastoma cells. ${ }^{41}$ Collectively, several studies indicate that targeting Rac1 may be associated with a therapeutic benefit, although Rac1 is also involved in physiological events such as vessel permeability indicating that targeting Rac1 could lead to leaky vessels. A detailed toxicological study must be conducted to evaluate which adverse effects are relevant in a therapeutic scheme with a Rac1 pharmacological inhibitor in vivo. These experiments are being conducted.

Taken together, this study provides evidence that Dock180/Rac1/Pak1 signaling pathway is downregulated by ZINC69391 compound leading to inhibition of cell proliferation and cell invasion on glioblastoma cell lines. Treatment with ZINC69391 arrested glioma cells in G1 phase, triggered apoptosis, and modulated actin cytoskeleton dynamics, resulting in inhibition of cancer cell motility. We also show that $1 \mathrm{~A}-116$ analog inhibits in vitro proliferation and invasion more efficiently. Our results encourage further preclinical testing in clinically relevant animal models.

\section{Acknowledgments}

We thank Dr Valeria Segatori for technical support in cytometry analysis. This work was supported by Consejo Nacional de Investigaciones Científicas y Técnicas (CONICET), Universidad Nacional de Quilmes, Agencia de Promoción Científica y Tecnológica (ANPCyT), Instituto Nacional de Tecnología Industrial (INTI), and by a grant from ChemoRomikin SA (Argentina).

\section{Disclosure}

The authors report no conflicts of interest in this work.

\section{References}

1. Maher EA, Furnari FB, Bachoo RM, et al. Malignant glioma: genetics and biology of a grave matter. Genes Dev. 2001;15(11):1311-1333.

2. Giese A, Bjerkvig R, Berens ME, Westphal M. Cost of migration: invasion of malignant gliomas and implications for treatment. $J$ Clin Oncol. 2003;21(8):1624-1636. 
3. Etienne-Manneville S, Hall A. Rho GTPases in cell biology. Nature. 2002;420(6916):629-635.

4. Rossman KL, Der CJ, Sondek J. GEF means go: turning on RHO GTPases with guanine nucleotide-exchange factors. Nat Rev Mol Cell Biol. 2005;6(2):167-180.

5. Bishop AL, Hall A. Rho GTPases and their effector proteins. Biochem J. 2000;348 Pt 2:241-255.

6. Fritz G, Just I, Kaina B. Rho GTPases are over-expressed in human tumors. Int J Cancer. 1999;81(5):682-687.

7. Salhia B, Tran NL, Chan A, et al. The guanine nucleotide exchange factors trio, Ect2, and Vav3 mediate the invasive behavior of glioblastoma. Am J Pathol. 2008;173(6):1828-1838.

8. Jarzynka MJ, Hu B, Hui KM, et al. ELMO1 and Dock180, a bipartite Rac1 guanine nucleotide exchange factor, promote human glioma cell invasion. Cancer Res. 2007;67(15):7203-7211.

9. Vigil D, Cherfils J, Rossman KL, Der CJ. Ras superfamily GEFs and GAPs: validated and tractable targets for cancer therapy? Nat Rev Cancer. 2010;10(12):842-857.

10. Cardama GA, Comin MJ, Hornos L, et al. Preclinical development of novel Rac1-GEF signaling inhibitors using a rational design approach in highly aggressive breast cancer cell lines. Anticancer Agents Med Chem. 2014;14(6):840-851.

11. Du J, Xu R, Hu Z, et al. PI3K and ERK-induced Rac1 activation mediates hypoxia-induced HIF-1alpha expression in MCF-7 breast cancer cells. PloS One. 2011;6(9):e25213.

12. Pille JY, Denoyelle C, Varet J, et al. Anti-RhoA and anti-RhoC siRNAs inhibit the proliferation and invasiveness of MDA-MB-231 breast cancer cells in vitro and in vivo. Mol Ther. 2005;11(2):267-274.

13. Wu X, Ramachandran S, Lin MC, Cerione RA, Erickson JW. A minimal Rac activation domain in the unconventional guanine nucleotide exchange factor Dock180. Biochemistry. 2011;50(6):1070-1080.

14. Ye DZ, Field J. PAK signaling in cancer. Cell Logist. 2012;2(2): $105-116$.

15. Aoki H, Yokoyama T, Fujiwara K, et al. Phosphorylated Pak1 level in the cytoplasm correlates with shorter survival time in patients with glioblastoma. Clin Cancer Res. 2007;13(22 Pt 1):6603-6609.

16. Olson MF, Ashworth A, Hall A. An essential role for Rho, Rac, and Cdc42 GTPases in cell cycle progression through G1. Science. 1995;269(5228):1270-1272.

17. Telford WG, King LE, Fraker PJ. Comparative evaluation of several DNA binding dyes in the detection of apoptosis-associated chromatin degradation by flow cytometry. Cytometry. 1992;13(2):137-143.

18. Senger DL, Tudan C, Guiot MC, et al. Suppression of Rac activity induces apoptosis of human glioma cells but not normal human astrocytes. Cancer research. 2002;62(7):2131-2140.

19. Chan AY, Coniglio SJ, Chuang YY, et al. Roles of the Rac1 and Rac3 GTPases in human tumor cell invasion. Oncogene. 2005;24(53): 7821-7829.

20. Ridley AJ. Rho GTPases and cell migration. J Cell Sci. 2001;114(Pt 15): 2713-2722.

21. Krauthammer M, Kong Y, Ha BH, et al. Exome sequencing identifies recurrent somatic RAC1 mutations in melanoma. Nat Genet. 2012;44(9): 1006-1014.

22. Hodis E, Watson IR, Kryukov GV, et al. A landscape of driver mutations in melanoma. Cell. 2012;150(2):251-263.

23. Fortin SP, Ennis MJ, Savitch BA, et al. Tumor necrosis factor-like weak inducer of apoptosis stimulation of glioma cell survival is dependent on Akt2 function. Mol Cancer Res. 2009;7(11):1871-1881.

24. Hwang SY, Jung JW, Jeong JS, et al. Dominant-negative Rac increases both inherent and ionizing radiation-induced cell migration in $\mathrm{C} 6$ rat glioma cells. Int J Cancer. 2006;118(8):2056-2063.
25. Yoon CH, Hyun KH, Kim RK, et al. The small GTPase Rac1 is involved in the maintenance of stemness and malignancies in glioma stem-like cells. FEBS Lett. 2011;585(14):2331-2338.

26. Vader P, van der Meel R, Symons MH, et al. Examining the role of Rac1 in tumor angiogenesis and growth: a clinically relevant RNAimediated approach. Angiogenesis. 2011;14(4):457-466.

27. Ma J, Xue Y, Liu W, et al. Role of activated rac1/cdc42 in mediating endothelial cell proliferation and tumor angiogenesis in breast cancer. PloS One. 2013;8(6):e66275.

28. Karnoub AE, Worthylake DK, Rossman KL, et al. Molecular basis for Rac1 recognition by guanine nucleotide exchange factors. Nat Struct Biol. 2001;8(12):1037-1041.

29. Gao Y, Xing J, Streuli M, Leto TL, Zheng Y. Trp(56) of rac1 specifies interaction with a subset of guanine nucleotide exchange factors. $J$ Biol Chem. 2001;276(50):47530-47541.

30. Feng H, Hu B, Vuori K, et al. EGFRvIII stimulates glioma growth and invasion through PKA-dependent serine phosphorylation of Dock180. Oncogene. 2014;33(19):2504-2512.

31. Feng H, Hu B, Liu KW, et al. Activation of Rac1 by Src-dependent phosphorylation of Dock180(Y1811) mediates PDGFR $\alpha$-stimulated glioma tumorigenesis in mice and humans. J Clin Invest. 2011;121(12): 4670-4684.

32. Kumar R, Gururaj AE, Barnes CJ. p21-activated kinases in cancer. Nat Rev Cancer. 2006;6(6):459-471.

33. Manser E, Leung T, Salihuddin H, Zhao ZS, Lim L. A brain serine/ threonine protein kinase activated by Cdc42 and Rac1. Nature. 1994; 367(6458):40-46.

34. Ong CC, Jubb AM, Haverty PM, et al. Targeting p 21-activated kinase 1 (PAK1) to induce apoptosis of tumor cells. Proc Natl Acad Sci U S A. 2011;108(17):7177-7182.

35. Park CM, Park MJ, Kwak HJ, et al. Ionizing radiation enhances matrix metalloproteinase-2 secretion and invasion of glioma cells through $\mathrm{Src} /$ epidermal growth factor receptor-mediated p38/Akt and phosphatidylinositol 3-kinase/Akt signaling pathways. Cancer Res. 2006;66(17): 8511-8519.

36. Wild-Bode C, Weller M, Rimner A, Dichgans J, Wick W. Sublethal irradiation promotes migration and invasiveness of glioma cells: implications for radiotherapy of human glioblastoma. Cancer Res. 2001;61(6):2744-2750.

37. Fortin Ensign SP, Mathews IT, Symons MH, Berens ME, Tran NL. Implications of Rho GTPase Signaling in Glioma Cell Invasion and Tumor Progression. Front Oncol. 2013;3:241.

38. Hirata E, Yukinaga H, Kamioka Y, et al. In vivo fluorescence resonance energy transfer imaging reveals differential activation of Rho-family GTPases in glioblastoma cell invasion. J Cell Sci. 2012; 125(Pt 4):858-868.

39. Hu B, Symons M, Salhia B, et al. Rho GTPases and Their Activators, Guanine Nucleotide Exchange Factors (GEFs): Their Roles in Glioma Cell Invasion. In: Fatatis A, editor. Signaling Pathways and Molecular Mediators in Metastasis. Springer Netherlands; 2012:143-169.

40. Sooman L, Ekman S, Andersson C, et al. Synergistic interactions between camptothecin and EGFR or RAC1 inhibitors and between imatinib and Notch signaling or RAC1 inhibitors in glioblastoma cell lines. Cancer Chemother Pharmacol. 2013;72(2):329-340.

41. Karpel-Massler G, Westhoff MA, Zhou S, et al. Combined inhibition of HER1/EGFR and RAC1 results in a synergistic antiproliferative effect on established and primary cultured human glioblastoma cells. Mol Cancer Ther. 2013;12(9):1783-1795. 
OncoTargets and Therapy

\section{Publish your work in this journal}

OncoTargets and Therapy is an international, peer-reviewed, open access journal focusing on the pathological basis of all cancers, potential targets for therapy and treatment protocols employed to improve the management of cancer patients. The journal also focuses on the impact of management programs and new therapeutic agents and protocols on

patient perspectives such as quality of life, adherence and satisfaction. The manuscript management system is completely online and includes a very quick and fair peer-review system, which is all easy to use. Visit http://www.dovepress.com/testimonials.php to read real quotes from published authors.

Submit your manuscript here: http://www.dovepress.com/oncotargets-and-therapy-journal 